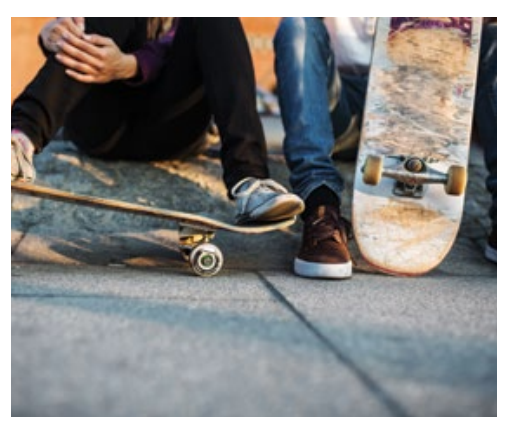

CPD

Lena Sanci, Marianne Webb, Jane Hocking

\section{Background \\ Adolescence is characterised by rapid changes in physical, cognitive, emotional and social development, and by experimentation and risk-taking, extending from the age of approximately 10 years into the early adult years. Developmental milestones achieved include a sense of identity, autonomy, a value system, peer relationships and financial independence. However, for around one in four adolescents, risk- taking and mental health issues threaten healthy biopsychosocial development. Such risks are rarely detected in clinical practice without deliberate assessment within a youth-friendly framework.}

\section{Objectives}

The aim of this paper is to provide an update on risk-taking behaviours in adolescence and highlight tips for re-orienting general practice towards youth-friendly preventive care for adolescents.

\section{Discussion}

General practice remains the health service most often accessed by young people and has important functions of engaging young people, providing positive experiences of healthcare, detecting risk and protective factors, and intervening early to promote healthy development. Future health system developments must ensure general practice has adequate time and support to provide effective preventive adolescent healthcare.

\title{
Risk-taking behaviour in adolescents
}

ADOLESCENCE is characterised by rapid changes in physical, cognitive and social development, beginning with puberty and ending in the acquisition of adult roles and responsibilities. In recent times, we have considered the developmental period of adolescence to extend from about 10 years of age into young adulthood, reflecting the longer time frames for developmental transition in four key areas: biological and sexual maturation, personal identity formation, engagement in intimate sexual relationships with an appropriate peer, and establishment of social independence and autonomy. ${ }^{1,2}$

The major influences shaping the social world of adolescents extend beyond the family to include peers, educational contexts, employment and the media. ${ }^{3}$ In our society, there are several periods of transition in social context that require adjustment during this time frame, from primary to secondary school and out of school to either employment or higher education, a combination of both, or neither.

With this emerging independence and new physical and cognitive abilities, adolescence is also characterised by experimentation and risk taking, sometimes with behaviours that may derail current and future health and wellbeing. Harms resulting from healthrisk behaviours are preventable, and if they have already occurred, early detection and intervention can reset trajectories and reduce ongoing harm. ${ }^{4,5}$
In countries such as Australia, which has an ageing population, we cannot afford the 'loss of human potential' arising from preventable health problems emerging during adolescence and frequently co-occurring, including consequences of tobacco, alcohol and substance use, unprotected sex, poor nutritional intake, low levels of exercise, injury, violence and mental disorders. ${ }^{5}$ The economic benefits of investing in the healthy development of adolescents for their current, future adult and offspring's health is now recognised. ${ }^{5}$

The World Health Organization has called for universal access to high-quality and preventive healthcare for all young people. ${ }^{3,6} \mathrm{High}$-quality healthcare services will engage adolescents effectively by being sensitive to the major developmental transitions they are undergoing alongside the greater propensity for experimentation. ${ }^{7}$

Given that $80 \%$ of adolescents consult general practice at least once per year, Australian general practice has an opportunity to meet the challenge of addressing risk-taking behaviours of adolescents with reference to current evidence-based guidance for preventive care during adolescence in The Royal Australian College of General Practitioners' Guidelines for preventive activities in general practice (Red Book). 8,9 This paper will provide an overview of risk-taking behaviours in adolescents and the general practice approach to preventive healthcare. 


\section{Health-risk behaviours and states}

\section{Accidents and injury}

In Australia, road accidents, suicides and self-inflicted injuries account for two-thirds of the injury burden for males and females aged $15-24$ years. ${ }^{10}$ Violence and homicide also rank highly, accounting for $6 \%$ of the male injury burden and $9 \%$ of the female injury burden. ${ }^{10}$ The total injury burden for young males is more than three times that for young females. ${ }^{10}$ The leading cause of death for young people of both sexes aged 15-24 years is suicide $(33 \%$ of all deaths in young people), followed by traffic accidents and accidental poisoning. ${ }^{11}$ Young drivers are over-represented among those killed and injured in road traffic accidents, attributed to a combination of inexperience and risky driving behaviours such as speeding, driving when tired, driving under the influence of alcohol or drugs, carrying passengers and using mobile phones when driving. ${ }^{12}$ Our work has revealed that engaging in road risks was strongly associated with other health-risk behaviours including tobacco, alcohol and illicit drug use and unprotected sex. ${ }^{12}$

\section{Mental disorders and self-harm}

One in four young people will have a mental disorder at any point in time, most commonly anxiety, depression and substance use disorders. ${ }^{13,14}$ More than $50 \%$ of adult mental disorders begin by age 14 years, and $75 \%$ begin by age 24 years, ${ }^{15}$ although not all mental health problems occurring during adolescence will progress to adult mental disorders, indicating the importance of early intervention. $^{3}$

Approximately one in 10 (10.9\%) young Australians aged 12-17 years reported having ever deliberately harmed themselves, three-quarters of whom self-harmed within the previous 12 months. ${ }^{13}$ Self-harm is associated with being female, depressive symptoms, anti-social behaviour and alcohol use. ${ }^{16}$ While not the only disorder associated with self-harm in adolescence, borderline personality disorder (BPD) is often diagnosed late, despite usually emerging between puberty and young adulthood, with a prevalence of $1-4 \% .{ }^{17}$ Recent calls for early intervention in BPD are based on well-established evidence that the disorder can be reliably diagnosed in adolescence and carries one of the highest burdens of disease, and new evidence that structured treatments are effective and that late intervention reinforces disability and the perception that no treatments will work. ${ }^{17}$ Recent analysis of emergency department attendances by Victorian children and adolescents (aged 0-19 years) revealed that, in contrast to a $13 \%$ rise in physical presentations, there was an alarming $46 \%$ rise in the annual number of those presenting with mental health problems from 2008-09 to 2014-15, particularly for self-harm, psychoactive substance use, depression and behavioural disorders. Adolescents aged 10-19 years accounted for most of these presentations, with the greatest rise in presentations being in adolescents aged 10-14 years. ${ }^{18}$ While the prevalence of mental health issues overall has not changed in young people aged 4-17 years from the 1998 to the 2013 Australian Child and Adolescent Survey of Mental Health and Wellbeing, the prevalence of major depressive disorder in adolescents aged 12-17 years increased from $2.9 \%$ to $5 \% .{ }^{13}$ A NSW study of emergency department presentations in 2010-14 related to self-harm, suicidal ideation or intentional poisoning revealed the presentation rate was highest for patients aged 10-19 years across all years, but the rate had grown most rapidly for patients aged $10-14$ years at $13.8 \%$ per year; the average overall rise in presentations per year for patients aged 10-19 years was $27 \%$, with over half the presentations outside normal working hours (8.00 am to $6.00 \mathrm{pm}) .{ }^{19}$ These figures highlight the importance of early detection and intervention in primary care to support adolescents with emerging mental health issues, and their families, to contribute to prevention of crisis emergency admissions. The researchers reporting on these trends are currently undertaking further study with families of adolescents presenting for mental health emergency care, and with healthcare providers, to increase understanding of the drivers and potential solutions.

\section{Sexual activity}

In Australia, the median age of first sex is 17 years. The last National Survey of Australian Secondary Students and Sexual Health of 2000 students in years 10-12 (aged approximately $15-18$ years) found that condoms were not used by $41 \%$ of participants the last time they had sex, placing them at risk of sexually transmissible infections (STIs). ${ }^{20}$ STIs have dramatically increased in Australia, with more than $50 \%$ of chlamydia cases and $30 \%$ of gonorrhoea cases occurring in people aged $<25$ years. ${ }^{21}$ STIs have major consequences, with $10 \%$ of chlamydia infections in women progressing to pelvic inflammatory disease (PID) and risk of tubal damage; $45 \%$ of tubal factor infertility is due to chlamydia. ${ }^{22}$

\section{Unintended pregnancy}

Most teen pregnancies in Australia are unintended and around half end in termination. ${ }^{23}$ The adolescent birth rate has declined in Australia over the past decade (10.5 births per 1000 females aged 15-19 years), but is still one of the highest in the Organisation for Economic Co-operation and Development. ${ }^{24}$ This may be because the most effective long-acting reversible contraceptives, which have $>99 \%$ efficacy at preventing pregnancy, were used by only $6 \%$ of young women in Australia. ${ }^{20}$

\section{Tobacco use}

Given that most adults who smoke begin during adolescence, it is important to prevent teenage smoking. ${ }^{25}$ Australian data from 2014 show that the prevalence of smoking is $10 \%$ in adolescents aged $16-17$ years and $3 \%$ in adolescents aged 12-15 years. This is a vast improvement since records began in 1984, when the prevalence of teenage smoking was $30 \%$ in adolescents aged $16-17$ years and $20 \%$ in adolescents aged $12-15$ years. National tobacco campaigns and strategies, smoke-free environments, large tobacco excises, plain packaging 
and media campaigns have all been operating over this time.

\section{Alcohol use \\ There are many reasons to assist adolescents to minimise harms from alcohol use. Alcohol is toxic to the developing brain, which is still refining reasoning and self-regulatory functioning into early adulthood; alcohol also impairs judgement and lowers inhibitions. . $26^{3}$ Improvements have been seen in teen drinking patterns, with the proportion of adolescents aged 12-17 years who abstain from alcohol significantly increasing from $72 \%$ in 2013 to $82 \%$ in $2016 .{ }^{27}$ Even young adults aged 18-24 years have reduced alcohol consumption, with the percentage exceeding lifetime risk guidelines declining from $31 \%$ in 2010 to $18.5 \%$ in 2016. However, in 2016 over $15 \%$ of young adults aged 18-24 years consumed alcohol at heavy levels ( $\geq 11$ drinks per occasion) at least monthly, with $5.3 \%$ needing medical attention and $2.5 \%$ admitted to hospital for an alcohol-related injury. ${ }^{26,27}$ Alcohol use is also implicated in unwanted sexual intercourse for $49 \%$ of young people. ${ }^{20}$ One in $10(10 \%)$ secondary school students report drinking alcohol weekly or more frequently. ${ }^{20}$}

\section{Drug use}

In 2016 , around $8.8 \%$ of adolescents aged $12-17$ years and $28.2 \%$ of young adults aged 18-24 years reported illicit drug use (eg cannabis, meth/amphetamines, ecstasy, cocaine).$^{27}$ The proportion of illicit drug use among adolescents aged 14-19 years has been decreasing since 2001, and people are initiating drug use later in life, with some commencing in their 40s. The most common drugs used within the past 12 months are cannabis and painkillers/opiates, especially overthe-counter codeine products, which have now been removed in Australia.

\section{Nutrition and exercise}

Nutrition is one of the most important behavioural risk factors for noncommunicable diseases; healthy dietary habits established in childhood and adolescence are likely to persist into adulthood. ${ }^{28}$ About one in three people aged 12-24 years met the 2003 National Health and Medical Research Council guidelines on daily fruit consumption (three serves per day for people aged 12-17 years and two for those aged 18-24 years), with males consuming less fruit than females. About one in seven people aged 12-17 years (14\%) met the guideline of four serves of vegetables per day, and only $5.4 \%$ of people aged 18-24 years met the guideline of five serves of vegetables per day.

Physical activity is also vital for the prevention of non-communicable diseases and improvement of psychosocial health and wellbeing in adolescence through better self-esteem, energy levels, sleep and concentration. ${ }^{29,30}$ For health benefits, young people aged 13-17 years are advised to accumulate at least 60 minutes of moderate-to-vigorous physical activity every day and do muscle and bone strengthening activities on at least three days per week. In 2011-12, about one in four young people ( $27 \%$ of people aged 12-24 years) were sedentary, with proportions greater among females, when compared with males (31\% versus $24 \%$ ). ${ }^{31}$

Australian data show $22 \%$ of young people are overweight and $15 \%$ are obese. $^{32}$ Being obese or overweight can negatively affect young people's wellbeing and increase the risk of adult obesity, ${ }^{33}$ chronic conditions such as type 2 diabetes and cancer, ${ }^{34}$ and mood disorders. ${ }^{35}$

Eating disorders are a mental health disorder but can come to attention because of repetitive dieting or restrictive eating, changes in food preferences, binge eating, purging and excessive exercise. Eating disorders have a severe impact on current and future health and wellbeing, and are often comorbid with mood disorders and substance use. ${ }^{36}$ The prevalence of eating disorders, particularly binge eating, appears to be increasing. ${ }^{37}$ Eating disorders tend to be poorly detected in primary care, and the index of suspicion needs to be high when there are early warning signs such as symptoms of depression, anxiety, irritability, preoccupation with body shape, weight, food and activities relating to food, weight loss or fluctuations in weight. ${ }^{38}$

\section{Reorienting general practice towards preventive care for young people}

Despite their psychosocial disease burden, physical issues are the most common reasons for people aged 15-24 years to visit general practitioners (GPs). ${ }^{39}$ Adolescents welcome the opportunity to discuss health issues such as contraception, substance use and STIs with clinicians, and trust the clinician's advice. ${ }^{40}$ However, adolescents tend not to disclose risk-taking behaviours unless prompted..$^{41}$ This is partly due to well-documented perceived barriers in accessing care (Box 1). ${ }^{9}$ While most young people attend primary care at least once per year, ${ }^{9}$ adolescents have the lowest attendance rates of all age groups, 3,39 meaning that the opportunity for early intervention and preventive care must be taken when adolescents present to general practice for any reason.

\section{Box 1. Barriers for adolescents accessing healthcare services}

The 5 Cs:

- Cost of care

- Confidentiality - fear of a lack thereof

- Compassion - lack of non-judgemental clinicians with sensitivity to developmental stage

- Clinical skill - poor communication by clinician (eg use of jargon) and low evidence-based management of common problems such as depression and anxiety or requests for effective contraception

- Convenience - difficulty getting a timely appointment, inflexible appointment systems, waiting times, inadequate transport options, restrictions on when can make time to attend care independently

and the D:

- Developmental issues - embarrassment, poor self-identified needs, low knowledge of services, little experience with healthcare systems and expressing needs, low health literacy. 
The Red Book recommends preventive health checks on the basis of HEEADSSS as part of a routine health assessment with young people (Box 2). The HEEADSSS domains are also prompts to explore protective factors and strengths in a young person's life and how these can be maximised in interventions addressing any health risks. Our prior trial and systematic review revealed benefits in structured health assessments for increasing the detection of risks within general practice ${ }^{42}$ and reducing some risk behaviours (eg substance use, diet, sex, alcohol, helmet use and exercise), as GP detection was linked to preventive interventions such as counselling, motivational interviewing or provision of effective contraception. ${ }^{43}$

Structured health assessments must occur within a youth-friendly framework that respects the young person's right to privacy and to shared and informed decision making about management (Box 3). Therefore, a GP would seldom conduct a health assessment pertaining to personal or sensitive issues in the presence of a parent or guardian; instead, they would ask for time alone with the young person to explore these issues and discuss with them what they want to share when their parent or guardian returns to the room. A similar approach is suggested when young people present with intimate partners, so that any issues around safety in the relationship can be explored when the partner is out of the room. One randomised trial found that 20\% (161/800) of young adults aged 17-24 years attending 40 Victorian general practices reported ever being afraid of an intimate partner or family member. ${ }^{42}$

\section{Box 2. HEEADSSS assessment ${ }^{46}$}

- Home environment

- Education and employment

- Eating and exercise

- Peer-related Activities

- Drugs, tobacco and alcohol

- Sex and sexuality

- Suicide, depression and other mental health issues

- Safety from injury, violence, abuse, and safety precautions to reduce sun damage and vaccine preventable infections

\section{Challenges for general practice in providing preventive care, and potential solutions}

GPs and practice nurses have found these preventive care approaches (screening for multiple risks and responding) acceptable; however, barriers include consultation time, lack of reimbursement and training, skills and confidence in responding, and limited availability of specialist services. ${ }^{9}$
One advantage of general practice providing long-term care is that the HEEADSSS assessment does not need to be completed at once, and clinicians may choose to prioritise the welcoming aspect of their practice so that young people are encouraged to return to discuss any aspect of their health, including risk behaviours. Other suggestions have been made to facilitate preventive care, such as a Medicare Benefits Schedule item number

\section{Box 3. A developmentally appropriate approach for youth-friendly preventive care consultations*}

\section{Introduction, welcome, explain process, empower, validate}

- Welcome and introduce yourself to the young person and show them where to sit.

- Validate concerns of the young person and parent/guardian if they attend.

- Acknowledge and praise help seeking, reassure that you can help.

- Negotiate time alone during the consult with the young person if they attend with a parent/guardian/intimate partner.

- Usually avoid asking personal/sensitive questions in front of parents/guardians/partners.

- If friends have been brought along by the young person for support, check if the young person is comfortable for their friends to stay and hear about their personal life.

\section{Explain confidentiality and signpost a preventive care psychosocial assessment}

- Discuss confidentiality and its exceptions (ie risk of serious harm including suicide/ homicide/sexual and physical abuse).

- Assess presenting issue, elicit concerns/fears.

- Explain the process of the consult (history/examination if appropriate/feedback/plan).

- Explain the purpose of questions about personal life and lifestyle (ie to check if any lifestyle behaviours are affecting health and wellbeing).

- Ask permission before asking personal/sensitive lifestyle questions.

- Give permission to decline to answer any of these personal questions.

Conduct a psychosocial risk and protective factor assessment

- Conduct the HEEADSSS risk and protective factor assessment.

- Remember to also ask about/comment on what is going well (strengths).

- Make your judgement about their overall level of psychosocial risk (eg low, low-medium but vulnerable, troubled, out of control).

- Provide feedback to the young person on aspects of their life you have assessed as going well and aspects needing further work. Praise their engagement in the process.

\section{Make a shared management plan}

- Negotiate a management plan with the young person: short, medium and long term.

- Assess the young person's capacity to consent to the management plan if they are under 18 years; document mature minor status.

- Rehearse with the young person what might be discussed with parents/guardians/others in young person's social context so that appropriate support can be provided.

- Provide education if required about the health issue and ongoing care.

- Use preventive care toolkit (eg motivational interviewing approaches, health promotion).

- Follow up as required, especially after referral for counselling, to ensure they have engaged, and for young people assessed as vulnerable and in need of risk monitoring.

- Instil a sense of hope.

* Refer to Adolescent health GP resource kit, 2 nd edition ${ }^{47}$ for more detail on steps such as the confidentiality discussion and health assessment. 
to allow appropriate time and remuneration for undertaking health assessments by either a GP or practice nurse. ${ }^{42}$

Our team has worked on an online health assessment tool (Check Up GP), co-designed with young people, parents, clinicians and reception staff, for young people to complete in around eight minutes before their consultations. ${ }^{44}$ Clinicians' view of the health assessment shows the HEEADSSS areas annotated by a traffic light system, with areas needing further assessment highlighted in red. Management tools and resources are also hyperlinked for the clinician to reduce their potential concerns about how to respond. Young people report preferring this online method of risk assessment, and clinicians also find it acceptable and efficient; however, they still need to manage time by prioritising risks to be assessed in one consultation and following up for others.

Finally, a pilot study in 100 Victorian secondary schools of a GP and practice nurse providing school-based primary care $^{45}$ showed that this system allowed more time to be spent with each adolescent to conduct a health-risk assessment within a youth-friendly care framework. If successful in meeting unmet preventive care and early intervention needs and in reducing pressure on local general practice and families, it may become a model worthy of expansion to address the growing mental health concerns and associated risks for this school-aged group.

\section{Conclusion}

Health-risk behaviours and mental health concerns peak in onset during adolescence and young adulthood and have deleterious but preventable consequences for current and future wellbeing. While public health initiatives have a role in reducing healthrisk behaviours, health services are also being called on to respond to individuals who often have complex and co-occurring problems. Early detection and intervention can make a difference in primary care, where most adolescents attend annually. Primary care requires time and support to undertake this important function. Further research will determine whether wide adoption of preventive general practice care for adolescence will help curb rising rates of crisis emergency department presentations for mental health and co-occurring issues.

\section{Authors}

Lena Sanci MBBS, PhD, FRACGP, Associate Professor, Department of General Practice, Melbourne Medical School; Faculty of Medicine, Dentistry and Health Sciences, University of Melbourne, Vic. I.sanci@unimelb.edu.au

Marianne Webb PhD scholar, Masters Youth Health \& Education Management, Department of General Practice, Melbourne Medical School; Faculty of Medicine, Dentistry and Health Sciences, University of Melbourne, Vic.webbm@unimelb.edu.au Jane Hocking BAppSc, MPH, MHlthSc, PhD, Professor, Melbourne School of Population and Global Health; Faculty of Medicine, Dentistry and Health Sciences, University of Melbourne, Vic. j.hocking@unimelb.edu.au

Competing interests: None.

Funding: MW has a part-time PhD scholarship from the Young and Well Cooperative Research Centre and $\mathrm{JH}$ is funded by a NHMRC Senior Research Fellowship.

Provenance and peer review: Commissioned, externally peer reviewed.

\section{Acknowledgements}

The authors would like to thank Dr Ann-Maree Duncan for administrative assistance with the references and box formatting.

\section{References}

1. Christie D, Viner R. ABC of adolescence: Adolescent development. BMJ 2005;330(7486):301-04. doi: 10.1136/ bmj.330.7486.301.

2. Sawyer $S M$, Afifi RA, Bearinger $L H$, et al. Adolescence: A foundation for future health. Lancet 2012;379(9826):1630-40. doi: 10.1016/ S0140-6736(12)60072-5.

3. Patton GC, Sawyer SM, Santelli JS, et al. Our future: A Lancet commission on adolescent health and wellbeing. Lancet 2016;387(10036):2423-78. doi: 10.1016/S0140-6736(16)00579-1.

4. McGorry PD, Purcell R, Hickie IB, Jorm AF. Investing in youth mental health is a best buy. Med J Aust 2007;187(7):S5-S7.

5. Patton GC, Olsson CA, Skirbekk V, et al. Adolescence and the next generation. Nature 2018;554(7693):458-66. doi: 10.1038/ nature25759.

6. World Health Organization. Health for the world's adolescents: A second chance in the second decade. Geneva: WHO, 2014.

7. Patton GC, Coffey C, Cappa C, et al. Health of the world's adolescents: A synthesis of internationally comparable data. Lancet 2012;379(9826):1665-75. doi: 10.1016/S0140-6736(12)60203-7.

8. The Royal Australian College of General Practitioners. Guidelines for preventive activities in general practice. 9th edn. East Melbourne, Vic: RACGP, 2016

9. Tylee A, Haller DM, Graham T, Churchill R, Sanci LA. Youth-friendly primary-care services: How are we doing and what more needs to be done? Lancet 2007;369(9572):1565-73. doi: 10.1016/S0140-6736(07)60371-7.
10. Eldridge D. Injury among young Australians. Canberra: Australian Institute of Health and Welfare, 2008.

11. Australian Bureau of Statistics. Causes of death, Australia, 2015. Cat. no. 3303.0. Canberra: ABS, 2016. Available at www. abs.gov.au/AUSSTATS/abs@.nsf/ DetailsPage/3303.02015?OpenDocument [Accessed 23 October 2018].

12. Martin J, Kauer S, Sanci L. Road safety risks in young people attending general practice: A crosssectional study of road risks and associated health risks. Aust Fam Physician 2016;45(9):666-72.

13. Lawrence D, Johnson S, Hafekost J, et al. The mental health of children and adolescents: Report on the second Australian Child and Adolescent Survey of Mental Health and Wellbeing. Canberra: Department of Health, 2015.

14. Patton GC, Coffey C, Romaniuk H, et al. The prognosis of common mental disorders in adolescents: A 14-year prospective cohort study. Lancet 2014;383(9926):1404-11. doi: 10.1016/ S0140-6736(13)62116-9.

15. Kessler RC, Berglund P, Demler O, Jin R, Merikangas KR, Walters EE. Lifetime prevalence and age-of-onset distributions of DSM-IV disorders in the National Comorbidity Survey Replication. Arch Gen Psychiatry 2005;62(6):593-602. doi: 10.1001/ archpsyc.62.6.593.

16. Heerde JA, Toumbourou JW, Hemphill SA, Herrenkohl TI, Patton GC, Catalano RF. Incidence and course of adolescent deliberate self-harm in Victoria, Australia, and Washington State. J Adolesc Health 2015;57(5):537-44. doi: 10.1016/j. jadohealth.2015.07.017.

17. Chanen A, Sharp C, Hoffman P; Global Alliance for Prevention and Early Intervention for Borderline Personality Disorder. Prevention and early intervention for borderline personality disorder A novel public health priority. World Psychiatry 2017;16(2):215-16. doi: 10.1002/wps.20429.

18. Hiscock H, Neely RJ, Lei S, Freed G. Paediatric mental and physical health presentations to emergency departments, Victoria, 2008-15. Med J Aust 2018;208(8):343-48.

19. Perera J, Wand T, Bein KJ, et al. Presentations to NSW emergency departments with self-harm, suicidal ideation, or intentional poisoning, 2010-2014. Med J Aust 2018;208(8):348-53.

20. Mitchell A, Patrick K, Heywood W, Blackman P, Pitts M. 5th national survey of Australian secondary students and sexual health 2013. Melbourne: Australian Research Centre in Sex, Health and Society, La Trobe University, 2014.

21. Department of Health. National Notifiable Diseases Surveillance System. Canberra: DoH, 2018. Available at www9.health.gov.au/cda/source/ rpt_5_sel.cfm [Accessed 23 October 2018].

22. Price MJ, Ades AE, Soldan $K$, et al. The natural history of Chlamydia trachomatis infection in women: A multi-parameter evidence synthesis. Health Technol Assess 2016;20(22):1-250. doi: 10.3310/hta20220.

23. Marino JL, Lewis LN, Bateson D, Hickey M Skinner SR. Teenage mothers. Aust Fam Physician 2016;45(10):712-17.

24. Organisation for Economic Co-operation and Development. Fertility rates. France: OECD, 2018. Available at https://data.oecd.org/pop/fertilityrates.htm [Accessed 23 October 2018].

25. Greenhalgh EM, Bayly M, Winstanley MH. Prevalence of smoking-Teenagers. In: Scollo MM, Winstanley $\mathrm{MH}$, editors. Tobacco in Australia: Facts and issues. Melbourne: Cancer Council Victoria, 2017 
26. Bruner AB, Fishman M. Adolescents and illicit drug use. JAMA 1998;280(7):597-98.

27. Australian Institute of Health and Welfare. National drug strategy household survey 2016: Detailed findings. Canberra: AlHW, 2017.

28. Australian Institute of Health and Welfare. Nutrition. Canberra: AlHW, 2015. Available at http://analytics.aihw.gov.au/Viewer/ VisualAnalyticsViewer_guest.jsp?report Path=\%2FAIHF\%2FReleased Public\%2FYout h\%2FReports\&reportName $=$ YTH 2015 nutrition\&appSwitchappSwitche=true [Accessed 23 October 2018].

29. Department of Health. Australia's physical activity and sedentary behaviour guidelines. Canberra: DoH, 2017. Available at http://www.health.gov. au/internet/main/publishing.nsf/content/healthpubhlth-strateg-phys-act-guidelines [Accessed 23 October 2018].

30. Okely AD, Salmon J, Vella $S$, et al. A systematic review to update the Australian physical activity guidelines for children and young people. Canberra: Department of Health, 2012.

31. Australian Institute of Health and Welfare. Level of physical exercise. Canberra: AlHW, 2015. Available at http://analytics.aihw.gov. au/Viewer/VisualAnalyticsViewer guest. jsp? reportPath=\%2F AlHF\%2FReleas edPubli c\%2FYouth\%2FReports\&reportName $=$ YTH2015_ EXERCISE\&appSwappSwitcherDis=true [Accessed 23 October 2018].

32. Australian Institute of Health and Welfare. A picture of overweight and obesity in Australia 2017. Canberra: AlHW, 2017.

33. Patton GC, Coffey C, Carlin JB, et al. Overweight and obesity between adolescence and young adulthood: A 10-year prospective cohort study. J Adolesc Health 2011;48(3):275-80. doi: 10.1016/j. jadohealth.2010.06.019.

34. Biro FM, Wien M. Childhood obesity and adult morbidities. Am J Clin Nutr 2010;91(5):1499S1505S. doi: 10.3945/ajcn.2010.28701B.
35. Sanderson K, Patton GC, McKercher C, Dwyer T, Venn AJ. Overweight and obesity in childhood and risk of mental disorder: A 20-year cohort study. Aust N Z J Psychiatry 2011;45(5):384-92. doi: 10.3109/00048674.2011.570309.

36. Lewinsohn PM, Striegel-Moore RH, Seeley JR. Epidemiology and natural course of eating disorders in young women from adolescence to young adulthood. J Am Acad Child Adolesc Psychiatry 2000;39(10):1284-92. doi: 10.1097/00004583-200010000-00016.

37. Hay P, Girosi F, Mond J. Prevalence and sociodemographic correlates of DSM-5 eating disorders in the Australian population. J Eat Disord 2015;3(1):19. doi: 10.1186/s40337-0150056-0.

38. Eating Disorders Victoria. Eating Disorders Victoria website. Abbotsford, Vic: Eating Disorders Victoria, 2015. Available at www.eatingdisorders. org.au/eating-disorders/what-is-an-eatingdisorder/warning-signs-a-symptoms [Accessed 23 October 2018].

39. Booth ML, Knox S, Kang M. Encounters between adolescents and general practice in Australia. J Paediatr Child Health 2008;44(12):699-705. doi: 10.1111/j.1440-1754.2008.01409.x.

40. Klein J, Matos Auerbach M. Improving adolescent health outcomes. Minerva Pediatr 2002;54(1):25-39.

41. Kramer T, Garralda ME. Psychiatric disorders in adolescents in primary care. Br J Psychiatry 1998;173:508-13.

42. Sanci L, Chondros P, Sawyer S, et al. Responding to young people's health risks in primary care: A cluster randomised trial of training clinicians in screening and motivational interviewing. PLoS One 2015;10(9):e0137581. doi: 10.1371/journal. pone. 0137581.

43. Webb MJ, Kauer SD, Ozer EM, Haller DM, Sanci LA. Does screening for and intervening with multiple health compromising behaviours and mental health disorders amongst young people attending primary care improve health outcomes? A systematic review. BMC Fam Pract 2016;17(1):104. doi: 10.1186/s12875-016-0504-1.

44. Webb MJ, Wadley G, Sanci LA. Experiences of general practitioners and practice support staff using a health and lifestyle screening app in primary health care: Implementation case study. JMIR Mhealth Uhealth 2018;6(4):e105. doi: $10.2196 /$ mhealth.8778.

45. Department of Education and Training. Doctors in secondary schools. Melbourne: DET, 2017. Available at www.education.vic.gov.au/about/ programs/health/Pages/doctors-secondaryschools.aspx [Accessed 23 October 2018].

46. Klein DA, Goldenring JM, Adelman WP. Probing for scars: How to ask the essential questions. Contemporary Pediatrics 2014;31(1):16-28.

47. Chown P, Kang M, Sanci L, Newnham V, Bennett D. Adolescent health GP resource kit, 2nd edition. North Sydney, NSW: NSW Health, 2016. Available at www.health.nsw.gov.au/ kidsfamilies/youth/Pages/gp-resource-kit.aspx [Accessed 23 October 2018] 